\title{
Work Related Musculoskeletal Injuries in Sonographers and Providers: The Grand Challenge
}

Yusef Sayeed, MD, MPH, MEng ${ }^{1}$

Keziah Sully, MD2

Kristine Robinson $\mathrm{MD}^{3}$

${ }^{1}$ Assistant Professor, Director of Pain Medicine and Functional Rehabiliation Eglin Air Force Base, Intrepid Spirit Center, Assistant Professor, Department of Physical Medicine and Rehabilitation, Department of Family Medicine, Uniformed Services University of the Health Sciences, Bethesda, MD

2 Interventional Physiatrist, Veteran's Administration, Department of Physical Medicine and Rehabilitation, Biloxi, MS

${ }^{3}$ Veteran's Health Administration, Department of Emergency Medicine, Assistant Professor, Department of Emergency Medicine, West Virginia University, Morgantown, WV

\section{Correspondence:}

Yusef Sayeed MD

96MDG/AMDS SGPF(IWC)

Eglin AFB, FL 32542

Email: yusef.a.sayeed.civ@mail.mil

Financial disclosure statements have been obtained, and no conflicts of interest have been reported by the authors or by any individuals in control of the content of this article.

These views do not represent the views of the United States Air Force, the United States Veterans Health Administration or the United States Department of Defense

Work related musculoskeletal disorders (WRMSD) has been widely reported among several occupations, and has taken a toll amongst sonographers as well. WRMSD disorders among sonographers had an incidence of up to $90 \%$, with shoulder pain being the most prevalent complaint [1]. WRMSD are under-reported among the more experienced sonographers, as well as among those in training. Recent survey data among Emergency Medicine Ultrasound Fellows has indicated similar injury patterns among physician counterparts in the United States. In reviewing the United States Bureau of Labor Statistics database (2018 is the most recent dataset) for injury/illness of Diagnostic Medical Sonographers (29-2032) and summarizing the findings:

Number of lost time injury/illness cases for Diagnostic Medical Sonographers 
(29-2032) is relatively constant from 2014-2017 but with an increase in 2018. No useful rate data. As expected, primarily upper extremity sprain/strain/pain due to overexertion due to worker positioning or motion. High level of disability (lost days) relative to other occupations [2].

Understanding the etiology of WRMSD helps address and prevent the issue. The United States Occupational Safety and Health Administration (OSHA) recognizes repetitive motions, bending, reaching overhead and working in awkward positions as known risk factors for musculoskeletal disorders, increasing the risk for a WRMSD. When these strains are not accommodated, WRMSD manifest as tendonitis, sprains, strains, bursitis, spondylosis, degenerative disc disease, and arthralgias. Continued repetition with relatively little recovery time results in chronic injury patterns. In fact, it has long been established that the primary perpetuating factor for WRMSD in the profession is poor positioning. Due to health care economics there has been a shift to increased productivity with sonographers having to provide increased exams with less rest cycles and decreased time to ergonomically accommodate the examination room [3].

There has long been a discussion both within the United States and Europe on how to best address these disorders. In fact the United States Centers for Disease Control and Prevention National Institute of Occupational Safety and Health has published guidelines to reduce WRMSD [4]. However, despite these best efforts there has not been a change in the injury patterns in the United States. The growing concern as the point of care ultrasound model continues to build these injury patterns will translate to our physician and provider counterparts in multiple specialties.

WRMSD has contributed to both personal and occupational impairment and disability for both individuals and employers including pain, difficulties with ADLs, lost work time (both absenteeism and presenteeism), costs incurred from lost work time (training/retraining, short term coverage employees, workers compensation insurance premium changes, and loss of productivity) and refractory cases have resulted in early retirements. Employer related costs, from both direct and indirect measures, has been estimated to be 120 billion US dollars each year. In fact, just absenteeism and lost work time alone cost employers approximately $\$ 21153 /$ week according to Roll et al. [5]. Recognition of this condition is vital, as it can only be prevented and addressed once it is acknowledged and prioritized. In this role, multiple national and international organizations and stakeholders have come together to form the 2020 Grand Challenge "Stop Work Related Musculoskeletal Disorders (WRMSD) Resulting from the Performance of Diagnostic Medical Ultrasound.“ [6]

This Grand Challenge has been designed with a grass roots initiative among stakeholders with efforts across three main pillars: research, education, and advocacy. It has brought together leading experts in the field of sonography, manufacturing, accreditation, occupational medicine, regulatory agencies, and those stakeholders who can best advocate for change at both the national and international level. This effort will require a multifaceted approach, much like what the nursing industry was able to do with safe patient handling initiatives. This international effort will join part- ners including the World Federation of Ultrasound in Medicine and Biology, the American Institute of Ultrasound in Medicine, the American Registry for Diagnostic Medical Sonography, the American College of Occupational and Environmental Medicine etc. ... with the hopes of changing the culture, work environment, applied ergonomics, and features of hazard controls so that our friends, colleagues, and peers continue to practice what they love and continue to make a difference in our patients lives.

\section{References}

[1] Murphy, Susan. Work Related Musculoskeletal Disorders in Sonography, SDMS White Paper. https://www.sdms.org/docs/default-source/ Resources/work-related-musculoskeletal-disor ders-in-sonography-white-paper.pdf?sfvrsn=8, accessed February $29^{\text {th }} 2020$

[2] United States Bureau of Labor Statistics, Injury Illnesses and Fatalities database, Survey of Occupational Injuries and IIInesses Date. https:// www.bls.gov/iif/soii-data.htm/\#summary, Accessed February 29 2020

[3] Evans K, Roll S, Baker J. Work-related musculoskeletal disorders (WRMSD) among registered diagnostic medical sonographers and vascular technologists: a representative sample. J Diagn Med Sonogr 2009; 25: 287-299

[4] Prevention of Musculoskeletal Disorders in the Workplace. Occupational Safety and Health Administration 2016. https://www.osha.gov/ SLTC/ergonomics/. Accessed July 17, 2016

[5] Roll SC et al. Contribution of Positioning to Work-Related Musculoskeletal Discomfort in Diagnostic Medical Sonographers. Work 2014; 47: $253-260$

[6] Work Related Musculoskeletal Disorders Grand Challenge. https://www.ardms.org/wrmsdgrand-challenge/, American Registry for Diagnostic Medical Sonography. accessed February $29^{\text {th }} 2020$ 